\title{
Emerging Smart Meters in Electrical Distribution Systems: Opportunities and Challenges
}

\author{
Abouzar Estebsari, Enrico Pons, \\ Ettore Bompard \\ Department of Energy \\ Politecnico di Torino \\ Turin, Italy, 10129 \\ Email: \{abouzar.estebsari, \\ enrico.pons, \\ ettore.bompard\}@polito.it
}

\author{
Alireza Bahmanyar, Sadegh Jamali \\ Department of Electrical \\ Engineering \\ Iran University of Science and \\ Technology \\ Tehran, Iran, 1684613114 \\ Email: \{bahmanyar, \\ sjamali\}@iust.ac.ir
}

\author{
Edoardo Patti, Andrea Acquaviva \\ Department of Control and \\ Computer Engineering \\ Politecnico di Torino \\ Turin, Italy, 10129 \\ Email: \{edoardo.patti, \\ andrea.acquaviva\}@polito.it
}

\begin{abstract}
High penetration of variable and non-programmable distributed generation has brought new challenges to the power system operation and is highlighting the need of a smarter grid. One of the key requirements in this regard is developing and deploying smart metering systems in distribution networks. In this paper we present the actual situation in the Italian distribution networks and we discuss the opportunities and challenges of applying new metering systems and introducing a flexible, multi-utility, multi-service metering architecture. Some off-the-shelf or prototype smart meters, selected to be tested in an ongoing European project, named FLEXMETER, are presented.
\end{abstract}

Keywords-electrical distribution systems; internet of things; smart grid; smart meter.

\section{INTRODUCTION}

Emerging paradigms for operation, control and management of power systems, especially in distribution grids, are relying more on communication networks and advanced metering systems [1]. The smart meter is an advanced meter that measures energy related information, at either end users' consumption point or secondary substation, to provide added information to both the utility company - or distribution system operator (DSO) - and customers. In other words, smart meters are electronic measurement devices which can be used by the utilities to obtain information for billing purposes and also for monitoring, controlling and operating their electric systems remotely [2]. The information is also used for better understanding of the system status from operator perspective. The system status could include system contingencies, faults, and operational behavior of the system.

Italian distribution systems (DSs) were designed and developed before the great deployment of distributed generation (DG), when electricity production and grid control were mainly centralized [3]. The structure of the DSs is mainly unchanged and is organized as follows. A primary substation feeds, through HV/MV transformers, a certain number of MV feeders. The feeders can be constituted by overhead lines (mainly in the countryside) or by underground cables (mainly in urban areas). Each feeder provides power to 10 to 20 secondary substations, where MV/LV transformers feed the downstream LV network. After the first portion of MV feeder, previously described, the line is sectioned and the rest of the feeder is energized by another primary substation. The MV distribution network is a poorly meshed network, but it is always radially operated, thanks to open sectionalizing switches. The LV network, that transfers power from the $\mathrm{MV} / \mathrm{LV}$ substations to the final customers, is also always radially operated, even if in some cases there are some ways of manually re-energizing a portion of it from a different secondary substation in case of fault.

In order to control the normal operation status of the DS, DSO can usually rely only on the measurements present in the primary substation, at the beginning of each MV feeder. In fact, the HV/MV transformers, which are commonly equipped with automatic on load tap changers for MV voltage regulation, need information on the current magnitude. These measurements are also required by the protection systems installed at the head of the MV feeders. The first step towards the remote control of the DS was transmitting the measurements of the primary substation to the DSO's control room. Instead, usually, no remote measurements are available from $\mathrm{MV} / \mathrm{LV}$ substations.

Italy was one of the first countries to develop a smart metering infrastructure [4]. Enel, the main Italian DSO, deployed its smart meter to all its customers, and sold the meters and also their communications technology to other Italian DSOs. Italian smart meters use Power Line Carrier (PLC) technology to transfer smart meter data to the nearest data concentrator, located in the MV/LV substation which feeds the customer, and GSM technology to send the data from the concentrator to the DSOs' data centers [5]. Totally, around 30 million meters and 400,000 secondary substations concentrators were installed.

Large customers and producers are monitored with 15 minutes time intervals, while the meters of ordinary customers with lower contractual power aggregate the energy consumption data on a monthly basis and data are retrieved only monthly. The implemented PLC communication technology, in fact, does not allow more data traffic on the distribution network. It would be therefore impossible to retrieve data with 15 minutes time intervals from all the meters. The consumption data gathered from the meters in Italy is 
currently used only for billing purposes. There are mainly two ways for the user to access the data measured by the smart meter:

- Dialoguing with it through PLC. This is not actually possible now, but will probably be possible in the near future thanks to the Smart Info device, developed by Enel [6];

- Adding a device to the smart meter which reads the led blinks, and converts the blinking in a measurement of consumed energy (1000 blinks are equal to $1 \mathrm{kWh}$ consumed). Many devices are already available off the shelf for this purpose.

The previously described methods, however, can only provide the customer with some approximate quasi real-time data about his power consumption. No useful information is obtained for the monitoring and operation of the distribution network. The new metering systems must go much further to satisfy more objectives like providing detailed consumption information, enabling energy consumption scheduling, realtime electricity pricing and market efficiency improvement, better network management and maintenance, advanced outage management and $\mathrm{CO}_{2}$ emissions and pollutants reduction.

In this regard, several projects co-funded by the European Commission (EC) have been carried out proposing and deploying different system architectures for more exploitation of smart meters. FLEXMETER is one of the ongoing EU Horizon 2020 projects aiming at the development and demonstration of a flexible smart metering architecture using different types of off-the-shelf smart meters for different general purpose services (electricity, water, gas, district heating). In this paper, we will firstly discuss the applications and challenges of advanced metering systems in electricity distribution networks, and then we will introduce FLEXMETER as a system which would overcome the challenges and meet the requirements of the emerging smart grids.

\section{EMERGING METERING SYSTEMS}

One of the main differences of the smart grids with respect to traditional grids is the two way communication system, which can be supported by smart meters [7]. In case of emergency, smart grids should have self-healing capability while the traditional distribution systems require a manual restoration. New generation of smart meters are able to shed or reconnect some predefined amount of loads automatically and remotely. In this case, customers' appliances can be remotely monitored and controlled, which eventually serves a better demand management.

One of smart meters advantages relates to prompt outage reporting. Utilities want to be alerted as soon as an outage occurs in the power system in order to take restoration action quickly. Battery powered smart meters which are able to send signals in case of outage detection, can be utilized in faster outage management strategies.

Main functionalities of a typical smart meter can be summarized as data collection/recording two-way communication, programming capability, and load control. Some typical smart meters deployed by Enel are shown in Fig. 4.

Smart meters are also beneficial and crucial for the grids with distributed generation penetration. They can measure the bidirectional power flows between grid and prosumer (electricity customer-producer), enabling accurate monitoring and recording of the exchanging energy. Radio Frequency (RF) and Power Line Communication (PLC) are the two basic types of communication technologies used for smart meters. Depending on utilities' requirements for the existing grids or future plans, appropriate technology is applied [1]. With Radio Frequency technology, smart meter sends the measurement data to a data collector through a wireless system. Then, the data is analyzed by different algorithms for utility desired applications, such as billing or outage management. In PLC technology, power lines are used for transferring data which are collected by the smart meters to the utility central data base [8]. In some cases, a combination of the two technologies is also proposed [9].

\section{A. Applications for customers}

One of the most interesting benefits of smart metering for customers is to make them more energy savvy. Detailed consumption information will enable customers to make smarter choices about their energy consumption schedules. Having the recent increases in energy price, users can participate in demand side management (DSM) to reduce their energy expenses by shifting their high-load household appliances to off-peak hours. Real-time electricity pricing brings potential benefits to the demand side and enables consumers to optimally adjust their consumptions to minimize their electricity bill. In [10] a pricing method for DSM is proposed to encourage efficient energy consumption among users to achieve certain social objectives.

An obvious perquisite of an efficient DSM is an automatic billing system and a real time pricing scheme. In conventional systems, personnel should travel long distances and take the reading manually. However, by remote monitoring of electronic energy meters this process can be avoided. An example of a remote monitoring and billing system is presented in [11]. In [12] authors show how optimal real-time pricing and load management strategies can allow users to reduce their electricity bill.

Another application which can directly benefit the customers is the systems that can provide information about instantaneous power and total energy consumption. These data can be grouped in different ranges, "(hourly, daily, weekly, monthly)" aiming to make the user aware of its consumption. This requires a display, showing the information in a simple and easy to understand representation, allowing the consumer to interact in almost real time.

Non-intrusive appliance load monitoring (NIALM), or energy disaggregation, is another interesting application that aims to break down a household's aggregate electricity consumption into individual appliances. In this way, it will be possible to inform the customer of how much energy each appliance consumes to help them to better manage their 
consumption. Moreover, by determining the current time of use of each appliance, the NIALM would be able to inform the customer of the potential savings through deferring appliance use to a time of day when electricity is either cheaper or has a lower carbon footprint [13].

\section{B. Applications for DSO}

Advanced metering systems will allow the DSO to better manage and maintain its network, distribute the energy more efficiently and, at the same time, reduce its operating costs. Automatic billing systems will decrease bill queries, complaints, and site visits. Automatic Meter Reading (AMR) lets the distributer to read customer meter remotely and send accurate, timely bills without regular on-site meter readings. Although AMR technology proved to be initially attractive, however it does not address some major issues they need to solve, for example demand-side management. AMR's capability, due to its one-way communication, is restricted to reading meter data. AMR does not let utilities to perform real time pricing schemes or take corrective action based on the information received from the meters. Consequently, rather than investing in AMR, utilities moved towards Advanced Metering Infrastructure (AMI). AMI not only provides a twoway communication, but also makes some applications (e.g. demand side management) possible [3].

Energy theft is one of the most important concerns of DSOs, which induce a considerable loss of income. With the introduction of AMI to electrical power systems, a variety of solutions to energy theft detection have been proposed. In [14], authors provide a survey of existing energy-theft detection schemes and classify them into three categories, i.e., classification-based, state-based, and game theory-based.

One of the most interesting advantages of AMI is in the area of outage detection and fault location. Electrical distribution networks, due to their geographical dispersion, may be affected by various faults arising from different sources such as adverse weather conditions, vegetation growth or equipment failure. Almost $90 \%$ of all power outages and disturbances have their roots in the distribution network [3]. During service interruptions, DSOs try to quickly manage the faults and restore power to reduce the economic impact of power outages.

Outages are mainly caused by permanent short circuit faults. When a short circuit fault occurs, protective devices close to the fault will automatically isolate the faulted area. Therefore, the loads downstream of the protective devices will be in outage. To localize outages, distribution companies mostly depend on customer calls. They have a list of all the customers with their telephone number and geographical location. When a customer calls, an operator or a computer performs a trouble call analysis process to determine the upstream protective devices involved in fault clearing and finds the outage area. However, during the nights there would be a little number of customer calls which makes the outage location process difficult or impossible. Moreover, false or fake reports are hard to identify and the whole process is time consuming and inefficient. Advanced Metering Infrastructure (AMI) for processing smart meter data would be a new and interesting option for outage reporting, which overcomes the above mentioned problems. Using the communication among the customer and feeder meters with the control center it would be possible to monitor the de-energized meters and, consequently, determine the part of the network that is in outage. The outage data can be incorporated by geographic information system (GIS) data, to map the outage area in a fast and efficient manner.

While the outage mapping methods can find the deenergized area of the faulted network, there are some techniques which are able to locate the faults which caused the resulting outage situation [15], [16]. With Information on accurate fault location, DSO can expedite service restoration and reconfigure the network to reduce outage time and costs. Traditional fault location methods (e.g. the impedance based methods [15]) are based on the voltage and current measured at $\mathrm{HV} / \mathrm{MV}$ substation. However, the emerging measurements which will be installed all over the system, from substation down to the customer location are the key to improve the accuracy, robustness, and speed of the pervious fault location methods [17]. Examples of such measuring devices include, but not limited to, feeder relay, intelligent capacitor bank controllers, reclosers, power quality meters and low-cost optical current and voltage sensors [17]. In [18] authors compare different fault location methods and show how the voltage measurements along the feeder can help to improve a traditional impedance-based method.

\section{Challenges}

The first challenge in deployment of advanced metering infrastructure is from the economic perspective. The design, installation and maintenance of the AMI involve many issues and require several billion dollars of investment for deployment and maintenance. Table I presents the average Smart Grid cost per customer, based on budget information from U.S. utilities' applications for the U.S. Department of Energy's Smart Grid Investment Grant (SGIG) program funds. At the first glance, justifying such investment seems difficult. However, it is worth mentioning that grid modernization creates direct and indirect economic benefits for customers. So a cost-benefit analysis would be a reasonable starting point for the evolution of future measurement infrastructures. The benefits can be differentiated to direct and indirect ones. Direct benefits are those that could directly affect customers' bills, while the indirect benefits are in terms of reliability and environmental performance improvements, which will have future economic impacts. In [19], authors summarize the Net Present Value (i.e. present value of future benefits) of costs and benefits assuming a 13 -year project life, incorporating 3 years of implementation and 10 years of operation. They conclude that Smart Grid offers a favorable benefit-to-cost ratio between 1.5 and 2.6 to 1 , when considering both direct and indirect economic benefits.

In the path of smart grid, besides the economic challenges, several security and privacy concerns arise. By deployment of smart meters with two-way communication capabilities, customers work closely with the utility to manage energy usage. The information they share reveal customer habits and behaviors, how they consume energy, the presence of people at 
TABLE I. AVERAGE COST PER CUSTOMER BY SMART GRID COMPONENT [19]

\begin{tabular}{|l|l|l|}
\hline Smart Grid Component & \multicolumn{1}{|c|}{ Sample Size } & \multicolumn{1}{|c|}{$\begin{array}{c}\text { Average Cost per } \\
\text { Customer }\end{array}$} \\
\hline Smart Meter & 24 projects & $\$ 291.54$ \\
\hline Distribution Automation & 12 projects & $\$ 63.64$ \\
\hline
\end{tabular}

their residence, and the appliances which are in use, exposing them to privacy invasions [20]. Moreover, some customers might be unwilling to communicate their consumption data with their neighbors' meter [4]. On the other side, grid and customers which are connected over a vast network of computerized meters can become a vulnerable target for hackers. Hackers can manipulate their energy costs or generate fake energy meter readings [20].

In a smart grid view to foster and provide new general purpose services to end users, a reliable and scalable distributed software infrastructure is needed, as discussed in previous works [21]. In this view, one of the major challenges consists on making available (near-) real-time data from smart meters to all the actors that need such information to provide a service. Hence, the smart meter must become an Internet-ofThings device. On this premises, a publish/subscribe communication approach is required [22], so that different actors can access the same information coming from the smart meters (more in general from the smart grid) for different purposes without affecting others. Publish/subscribe is an event-based and data-centric communication model that allows the development of loosely-coupled event-based systems. This approach decouples the production and consumption of information by removing all the explicit dependencies between the interacting entities, which increases scalability. As example, Fig. 1 shows the publish/subscribe information flow, where three entities are involved: i) Message broker, ii) smart meter, iii) services. The Message Broker is a software component that forwards the information, sent by smart meter (the publisher), to the different services (the subscribers).

\section{Metering System ARCHITECTURE}

In this section, the already deployed metering system architecture in Italy is presented, and a flexible metering system proposed in the ongoing FLEXMETER project is introduced along with some smart meters selected to be exploited in its framework.

\section{A. Enel's metering system}

Regarding smart metering, Enel distribution system operator has deployed an innovative system called "Telegestore" to manage LV residential and commercial smart meters remotely [23]. Telegestore (as Enel Automatic Meter Management (AMM) solution) architecture (Fig. 2) consists of: integrated electronic meters with breakers (for measurements, contract management and PLC communication functions); AMM which talks with LV concentrators (CBT) in every MV/LV substation through GSM, PSTN and/or satellites as public communication networks; the LV Concentrator (LVC)

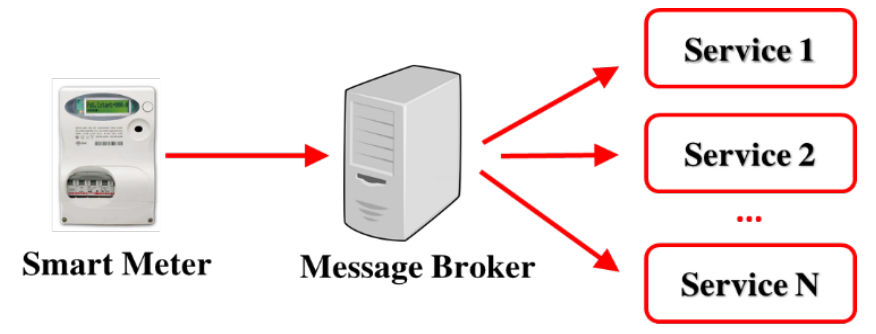

Figure 1. Publish/subscribe information flow for general purpose services

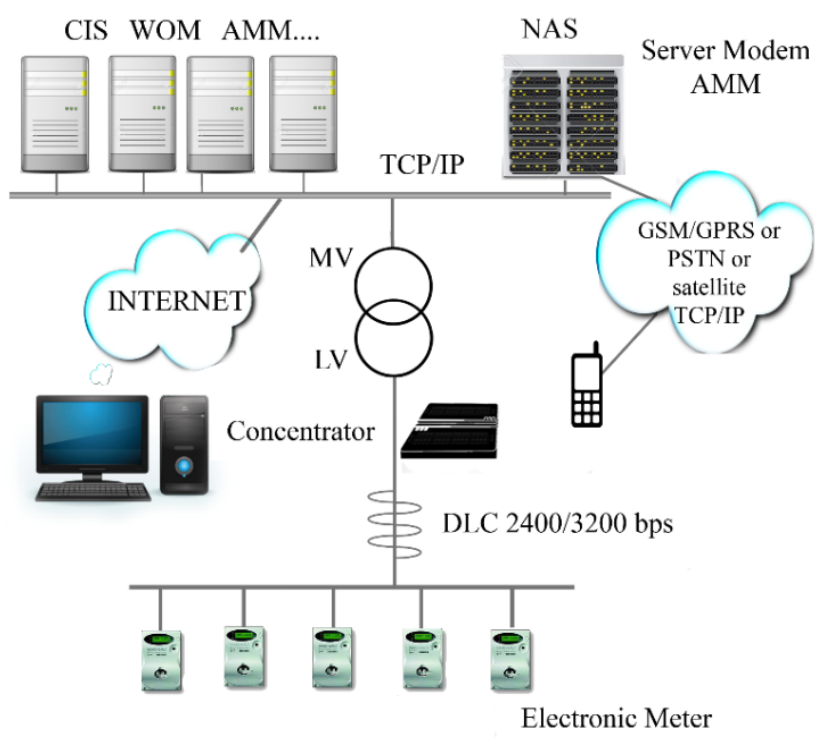

Figure 2. Telegestore system architecture

for managing the communication of the Remote Metering Central System and the meters; TCP/IP protocol for communication between AMM and concentrator (CBT).

Some of the main functionalities of Telegestore system are remote reading, remote load disconnecting/reclosing which enjoys the bidirectional communication capability of the installed smart meters, comparison between total energy delivered to consumers and energy downstream flow in $\mathrm{MV} / \mathrm{LV}$ substation, theft or fraud detection, active and reactive energy measurement with different time samples (from 1 minute to 1 hour), storing measurements of 15 minute sample time for 38 days, etc.

\section{B. FLEXMETER multi-service metering architecture}

The FLEXMETER project aims to develop and implement a flexible metering architecture based on a distributed software infrastructure that exploits publish/subscribe [22] and request/response communication models to access information from the smart meters. FLEXMETER also aims to deploy plug and play off-the-shelf meters [24]. These meters are for measuring different energy flows (e.g. electricity, gas, water) to provide advanced general purpose services for customers, DSOs and other utilities. The proposed architecture (Fig. 3) is planned to serve services like consumption historical data recording, demand side management, fault and outage management, energy theft detection, and network balancing. 
FLEXMETER system is enabled to collect and analyze the measurement data from different types of smart meters either from manufacture technology aspect or from installation point perspective. Some of the selected off-the-shelf meters are introduced herein.

The most typical and available meters are Enels which allow for a bidirectional communication with the control center: they can send the measured data while can receive new settings regarding tariffs and contractual power, and commands for disconnecting the customer.

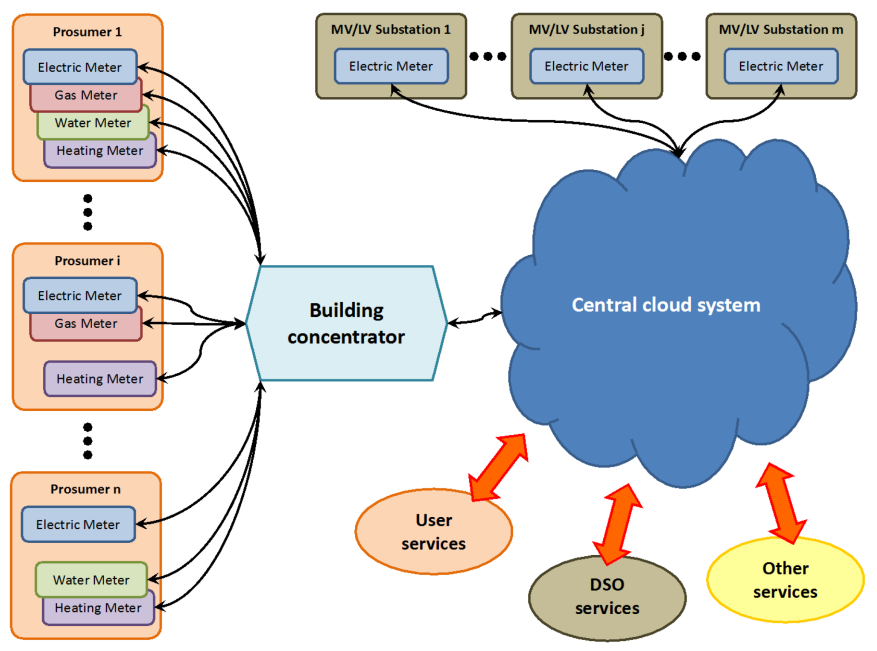

Figure 3. FLEXMETER Architecture

Fig. 4 shows three types of existing smart meters already installed at end-user customers. By simply pushing the button, customer local information is shown on the display of the meter. The remote operation like parameters set up, disconnection, and enabling command for locally manual connecting are all possible.

The general characteristics of these meters are active and reactive energy and power measurement, 15 years life-time, less than $0.3 \%$ failure rate, less than $2 \mathrm{~W} /$ phase power selfconsumption, suitable for environmental operational conditions in the range of $-25^{\circ} \mathrm{C}$ to $+55^{\circ} \mathrm{C}$ and limits of $-40^{\circ} \mathrm{Cand}+70^{\circ} \mathrm{C}$, and daily self-diagnostics on the main components and functions.

Energy-Neutral and Nonintrusive Wireless Energy Meter (NIWEM) is another type of smart meters which is full battery free and completely non-invasive smart meter which measures voltage, current and power factor. A standard wireless interface, based on ZigBee protocol, is used for communication with the smart-building system [25].

STCOMET (Fig. 5), a prototype meter manufactured by STMicroelectronics is a device that integrates a power line communication (PLC) modem, a high-performance application core and metrology functions. STCOMET simplifies the design

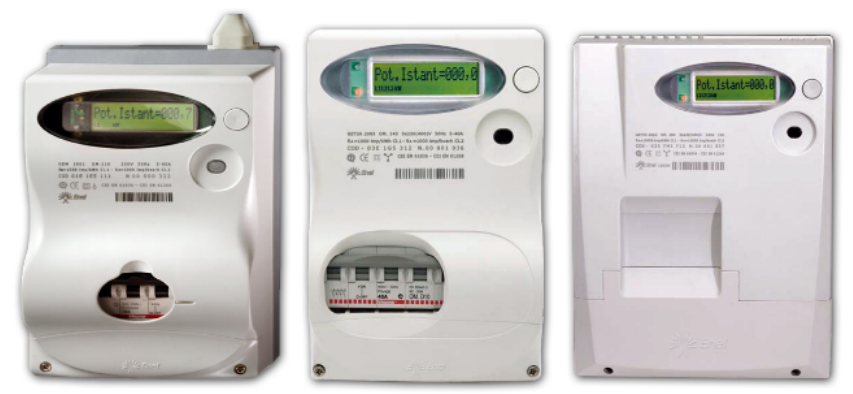

Figure 4. Smart meters used for Telegestore - from left to right: single phase (GISM), poly phase meter for demand up to $30 \mathrm{~kW}$ (GIST), poly phase meter with current transformer (CT) for demand up to $200 \mathrm{~kW}$ (GISS).

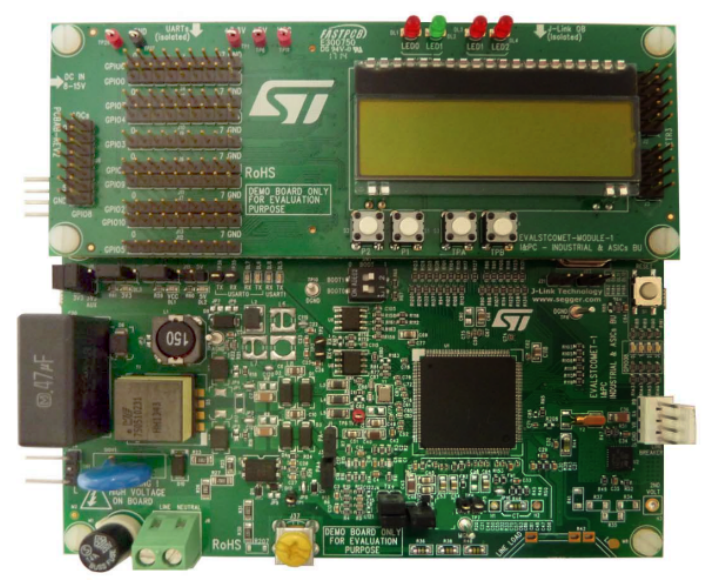

Figure 5. STCOMET platform development environment

of smart meters by implementing key functions on a single chip, including a PLC modem compliant with major smartmetering industry standards. STCOMET is intended to be installed on both LV network and MV/LV transformers.

The OpenEnergyMonitor.org system is an open-source monitoring system for electricity, temperature and humidity. The system architecture consists of 5 modules integrated for different applications from home energy monitoring to solar PV import/export monitoring [26]. The emonTx and emonPi with monitoring capability of maximum 4 and 2 single-phase $\mathrm{AC}$ circuits respectively, and using clip-on CT sensors, are mainly used for electricity measurements. A plug-in AC-AC adapter powers the unit and provides an AC voltage sample for Real Power calculations. emonTH is temperature and humidity sensor and emonBase is the internet gateway, relaying readings received via wireless from sensor nodes (emonTx / emonTH) to emoncms, an open-source energy monitoring webapplication. emonTx data is transmitted via low power $433 \mathrm{Mhz}$ RF back to emonPi or emonBase.

As one of the applications planned in FLEXMETER addresses the customer awareness, Smart Info, developed by Enel in July 2011, will be used as a Plug \& Play device which establishes a direct connection between the meter and the customer allowing them direct access to information recorded by the electronic meter. In other words, Smart Meter collects a 
large set of metering data, and then through Smart Info it is possible to select a preconfigured subset of the available data for the support new advances services, such as automatic control of loads, integration of smart appliances, enabling of real time tariffs and active demand services [6]

Other electricity related intended general purpose services of FLEXMETER framework, such as fault and outage management, and energy theft detection are under investigation to be implemented as well after complete architecture is deployed.

\section{CONCLUSION}

Smart metering not only provides huge advantages to utility companies as well as customers and other energy players, but also involves various potential challenges. Smart meters already installed in Italy are operated much below their potential capabilities, mainly because of the adopted communication technology. In the EU H2020 FLEXMETER project we aim to deploy plug and play off-the-shelf and prototype meters, and to develop and implement a flexible metering architecture based on a distributed software infrastructure. Many services will be implemented based on the installed meters and on the software infrastructure, including outage detection, fault location, user awareness and demand response.

\section{ACKNOWLEDGMENT}

This work was supported by FLEXMETER, which is a H2020 European Research Project (http://flexmeter.polito.it/).

\section{REFERENCES}

[1] J. Zheng, D.W. Goa and L. Lin , "Smart meters in smart grid: an overview" , Proc. IEEE Green Technologies Conference, pp. 57-64, April 2013.

[2] E. Queen, "A discussion of smart meters and RF exposure issues," Edison Electric Institute (EEI), Washington, D.C, A Joint Project of the EEI and AEIC Meter Committees, March 2011.

[3] H. Farhangi, "The path of the smart grid" , IEEE Power Energy Mag., vol. 8 , no. 1 , pp. 18-28, 2010.

[4] S. Depuru, W. Lingfeng, V. Devabhaktuni and N. Gudi "Smart meters for power grid Challenges, issues, advantages and status" IEEE PES Power Systems Conference and Exposition (PSCE), pp. 1-7, March 2011.

[5] V. Gungor, D. Sahin , T. Kocak , S. Ergut, C. Buccella , C. Cecati and G. Hancke, "Smart grid technologies: Communications technologies and standards" , IEEE Trans. Ind. Informat. , vol. 7 , no. 4 , pp.529 -539 , 2011.

[6] ENEL project, "Smart info +", Available at: http://eneldistribuzione.enel.it/it-IT/Pagine/enel_info_piu_sezione.aspx.

[7] X. Fang, S. Misra, G. Xue and D. Yang, "Smart grid - the new and improved power grid: A survey" , IEEE Commun. Surveys Tutorials , pp. 944-980, 2012.

[8] Smart Meters and Smart Meter Systems A Metering Industry Perspective, an EEI-AEIC-UTC White Paper, Published by Edison Electric Institute, Pennsylvania, 2011.
[9] M. Rafiei and S. M. Eftekhari, "A practical smart metering using combination of power line communication (PLC) and WiFi protocols," in Proceedings of 17th Conference on Electrical Power Distribution Networks (EPDC), 2012, pp. 1-5.

[10] P. Samadi , A. H. Mohsenian-Rad, R. Schober and V. W. S. Wong , "Advanced demand side management for the future smart grid using mechanism design", IEEE Trans. Smart Grid, vol. 3 , no. 3 , pp.1170 $1180,2012$.

[11] M. Islam, M. Ahmad, M. Islam, A. Mitul, M. Malek and M. Rashid, "Electronic energy meter with remote monitoring and billing system", 7 th International Conference on Electrical \& Computer Engineering (ICECE), pp. 240-243, Dec. 2012.

[12] Z. Chen, L. Wu and Y. Fu, "Real-time price-based demand response management for residentialappliances via stochastic optimization and robust optimization", IEEE Trans. Smart Grid, vol. 3 , no. 4 , pp.1822 $1831,2012$.

[13] O. Parson, S. Ghosh, M. Weal and A. Rogers , "Non-intrusive load monitoring using prior models of general appliance types", Proc. 26th Conference on Artificial Intelligence, pp.356 -362.

[14] R. Jiang , "Energy-theft detection issues for advanced metering infrastructure in smart grid" , Tsinghua Science and Technology, vol. 19 , no. 2 , pp.105 $-120,2014$.

[15] R. H. Salim , M. Resener , A. D. Filomena , K. R. Oliveira and A. S. Bretas , "Extended fault-location formulation for power distribution systems", IEEE Trans. Power Del., vol. 24, no. 2 , pp.508 -516, 2009.

[16] S. Jamali, A. Bahmanyar and H. Borhani-Bahabadi, "A Fast and accurate fault location method for distribution networks with DG using genetic algorithms" Smart Grid Conference (SGC), Dec. 2015.

[17] M. Kezunovic , "Smart fault location for smartgrids" , IEEE Trans. Smart Grid, vol. 2 , no. 1, pp.11 -22, 2011.

[18] S. Lotfifard, M. Kezunovic, and M. J. Mousavi, "A Systematic Approach for Ranking Distribution Systems Fault Location Algorithms and Eliminating False Estimates, " IEEE Trans. Power Del., vol. 28, no. 1, pp. 285-293, Jan. 2013.

[19] Smart Grid Consumer Collaborative, "Smart grid economic and environmental benefits", October 2013, Available at: http://smartgridcc.org/wp-content/uploads/2013/10/SGCC-Econ-andEnviron-Benefits-Full-Report.pdf.

[20] P. McDaniel and S. McLaughlin, "Security and privacy challenges in the smart grid" , IEEE Security \& Privacy, vol. 7 , no. 3 , pp. $75-77$ , 2009.

[21] E. Patti, A. Syrri, M. Jahn, P. Mancarella, A. Acquaviva and E. Macii , "Distributed software infrastructure for general purpose services in smart grid", IEEE Trans. on Smart Grid , pp.1 -8 , 2014.

[22] P. T. Eugster , P. A. Felber, R. Guerraoui and A.-M. Kermarrec , "The many faces of publish/subscribe" , ACM Comput. Surv., vol. 35 , no. 2 , pp.114 -131, 2003.

[23] B. Botte, V. Cannatelli, and S. Rogai, "The telegestore project in enel's metering system," 18th International Conference on Electricity Distribution, pp. 1-4, June 2005.

[24] E. Patti, E. Pons, D. Martellacci, F. Boni, A. Acquaviva, and E. Macii, "multiflex: Flexible multi-utility, multi-service smart metering architecture for energy vectors with active prosumers," 4th International Conference on Smart Cities and Green ICT Systems (SMARTGREENS 2015), Lisbon, Portugal, 20-22 May, 2015. pp. 288-293.

[25] D. Balsamo, G. Gallo, D. Brunelli and L. Benini , "Non-intrusive zigbee power meter for load monitoring in smart buildings" , Sensors Applications Symposium (SAS), 2015 IEEE , 2015.

[26] Open energy monitor, "open-source energy monitoring tool", Availabe at: 